\title{
La educación superior ante los desafíos sociales
}

\section{Higher education in the face of social challenges}

Estudios

Adrián Cuevas Jiménez

Universidad Nacional Autónoma de México - México cuevasjim@gmail.com

Recibido: 4 de enero de 2016 / Aceptado: 30 de junio de 2016

\section{Resumen}

La educación superior refiere a la formación educativa posterior al bachillerato, es decir licenciatura y posgrado, cuya misión es preservar, crear y difundir la cultura. A lo largo de la historia la educación superior ha vivido un proceso de trasformación, debido principalmente al desarrollo del conocimiento y a la trasformación de la sociedad. En ese proceso resaltan dos grandes momentos; en el primero, que culminó a mediados del siglo $X X$, se concebía que la institución de educación superior abarcaba todo el conocimiento de la sociedad, y que quien se graduaba ya estaba preparado para desempeñarse profesionalmente durante todo la vida; en el segundo momento, a partir de esas fechas, se concibe que los conocimientos ya no son exclusividad de la institución de educación superior, y no puede haber desempeño eficiente sin formación continua ni actualización permanente de conocimientos. El objetivo de este trabajo es explicitar las metas generales y algunas estrategias de formación de los estudiantes de educación superior, para afrontar los grandes desafíos que enfrenta hoy la sociedad. Para definir dichas metas y estrategias se consideran cuatro niveles de desafío: a) desafíos físicos, estructurales y político-ideológicos; b) desafíos en torno a los avances científico-técnicos y del conocimiento; c) desafíos de la estructura interna del proceso formativo y del acceso a la educación superior, y d) desafíos en la formación de valores en los estudiantes.

Palabras clave: Desafío social, educación superior, estrategia de formación.

\section{Abstract}

Higher education refers to the subsequent training to high school education, that is undergraduate and graduate, whose mission is to preserve, develop and disseminate culture. Throughout the history of higher education it has undergone a process of transformation, mainly due to the development of knowledge and the transformation of society. In the process they highlighted two great moments; in the first, which culminated in the mid-twentieth century, it conceived the higher education institution encompassing all knowledge of society, and who graduated was ready to perform professionally throughout life; the second time, after those dates, it is conceivable that knowledge is no longer exclusive to the institution of higher education, and there can be no efficient performance without continuous training and continuous updating of knowledge. The objective of this work is to point out the general goals and some strategies of the students' formation of superior education, to confront the big challenges that it faces today the society. To define this goals and strategies four challenge levels are considered: a) physical, structural and politicalideological challenges; b) challenges around the scientifictechnician and of the knowledge advances; c) challenges of the internal structure of the formative process and the access to the superior education, and d) challenges in the formation of values in the students.

Keywords: Social challenge, superior education, formation strategy.

Forma sugerida de citar: Cuevas Jiménez, Adrián (2016). La educación superior ante los desafíos sociales. Alteridad, 11(1), pp. 101-109. 


\section{Introducción}

De manera general, la educación superior hace referencia al nivel de formación educativa que es posterior al bachillerato, y que incluye tanto la licenciatura -a la que se suele asignar una formación profesional en las distintas áreas en las que se divide el conocimiento-, como el posgrado -cuya formación suele orientarse más hacia la investigación. Las instituciones que llevan a cabo la educación superior tienen el encargo social de preservar, crear y difundir la cultura, entendiendo por ésta toda la obra creada por el ser humano, es decir, el conjunto de conocimientos, técnicas, prácticas, creencias, valores, formas de acción, de relación y de comunicación generados. En el cumplimiento de esta encomienda y a partir de las necesidades sociales, económicas, culturales y de desarrollo científico-técnico de la sociedad en la que se insertan, dichas instituciones llevan a cabo una formación profesional y científica de pregrado y postgrado, en aras de conservar y producir conocimientos en las distintas áreas y esferas de la realidad, así como su difusión a los distintos ámbitos de la sociedad en la que se insertan. Se trata de garantizar la transferencia del acervo cultural a las nuevas generaciones a través de las actividades y prácticas de formación, desarrollar la cultura a través de la investigación científica, así como su promoción en la sociedad. Esa formación superior implica un conjunto de lineamientos, acciones, disposiciones y prácticas que sistematizan formalmente la preparación del profesional, que capacitan y legitiman el desempeño en las distintas profesiones y áreas en las que se distribuye el conocimiento y a través de las que se estructura dicho nivel formativo.

En torno a esa misión de la institución superior: preservación, desarrollo o creación y promoción de la cultura, existen posiciones diferentes en cuanto a las prioridades que al respecto se asumen; en algunos contextos se privilegia la preservación sobre los demás rubros, colocando el acento y los esfuerzos en el aspecto docente formativo; en otros se asumen como tres funciones independientes y/o separadas, y hay casos en los que se consideran como tres cuestiones primordiales y estrechamente vinculadas, concibiendo así que, formación, investigación y extensión constituyen los procesos esenciales de la institución superior, indisolublemente interrelacionados.

La educación superior ha experimentado, a lo largo de su historia, cambios y transformaciones, impulsados principalmente por el desarrollo del conocimiento y la transformación de la sociedad en la que se inscribe; en ese devenir se hace posible identificar al menos dos grandes momentos: el primero, con límites hasta mediados del siglo XX, estuvo caracterizado por concebir que el centro de educación superior era la institución que atesoraba todo el conocimiento de la sociedad $y$, asimismo, que quien culminaba una profesión estaba preparado de una vez y para siempre, para desempeñarse laboralmente para toda la vida; el segundo momento, a partir de esas fechas y principalmente en nuestros días, se caracteriza por la concepción de que ya nada sucede de esa manera, es decir, ni los conocimientos son una exclusividad de las instituciones de educación superior ni se puede concebir un desempeño eficiente y eficaz durante toda la vida laboral sin una permanente formación y actualización de conocimientos, y de ahí el planteamiento de la Organización de las Naciones Unidas para la Educación la Ciencia y la Cultura (UNESCO) (1992), de "educación para todos durante toda la vida" como caracterización de la educación para nuestro tiempo.

El objetivo de este trabajo es explicitar las metas generales y algunas estrategias de formación de los estudiantes de nivel superior, a partir de delinear algunos de los principales desafíos que se requiere afrontar, ante esa nueva perspectiva de la educación y ante las transformaciones tanto sociales como económicas, políticas, científico técnicas y físicas que se viven en la actualidad.

\section{Los principales desafíos de la sociedad}

Se trata de las condiciones y dilemas que en nuestros días presenta el mundo físico y social, así 
como los avances del conocimiento científico y técnico logrados por la humanidad, para cuyo afrontamiento es imprescindible el papel de las instituciones de educación superior; en ese mismo sentido se encuentran también las cuestiones que tienen que ver con los dilemas que genera la relación entre la formación superior y la formación previa a ella, con el acceso y la permanencia, y con la estructuración y relación interna del proceso formativo; finalmente, se trata también de los desafíos concernientes con la formación personal integral de los universitarios, a través de su formación en valores que los implique profesional y comprometidamente en la solución de los problemas y necesidades de su sociedad, anteponiendo el bienestar colectivo al interés personal.

\section{Desafíos físicos, estructurales y político-ideológicos}

La educación superior requiere tener en cuenta el contexto general en el que se sitúa, ya que debe afrontar sus necesidades y continuar sus logros culturales; los siguientes son algunos aspectos en este nivel.

Las condiciones físicas, socioeconómicas y político-ideológicas de la actualidad, constituyen el escenario que da cuenta de grandes transformaciones en torno a la realidad humana, entre las que sobresalen: a) la formación de nuevos valores; b) la caída de ideologías instituyentes de metas que con anterioridad dirigían las prácticas con algún grado de confianza y certeza hacia un fin seguro; c) el reconocimiento y respeto a la diversidad cultural para convivir en la diferencia; d) la toma de conciencia hacia los enormes problemas que, en aras del "desarrollo", han sido generados por el mismo hombre y que amenazan la existencia misma de la especie humana, como el deterioro ecológico, el calentamiento global y la escasez de recursos naturales, entre otros; asimismo, e) una situación general envolvente en la que, ante el gran avance de las comunicaciones, ya no existen lejanías posibles que vuelvan al individuo indiferente a cualquier suceso que ocurra en cualquier parte del planeta.
El mundo moderno es apocalíptico, no porque se encamine inevitablemente hacia la catástrofe, sino porque implica riesgos que las generaciones anteriores no tuvieron que afrontar(...) los riesgos de una catástrofe ecológica forman parte ineludible del horizonte de nuestra vida cotidiana. Otros riesgos de consecuencias graves, como el hundimiento de los mecanismos de la economía mundial o la aparición de superestados totalitarios, forman parte de nuestra experiencia contemporánea de manera igualmente ineludible (Giddens, 1995, pp. 12-13).

Es decir, se vive en la actualidad una realidad bastante compleja, colmada de grandes incertidumbres y retos, que exige -y condiciona a la vez, para desenvolverse en ella- una formación amplia que implica, entre otras cuestiones, el desarrollo de estrategias y habilidades para comprender el mundo, la formación de cualidades personales para tomar decisiones, para adoptar o crear un estilo de vida y para la disposición continua al cambio; como señalan algunos autores, situación de riesgo amenazante implica formarse y vivir en una actitud de cálculo continuo hacia las posibilidades de acción, favorables o desfavorables, con las que la existencia contemporánea se encuentra a diario en lo social, en lo físico, en lo individual y colectivamente (Giddens, 1995, Sotolongo, 2006; Morín, 2001). Consecuentemente, quienes se forman como profesionales, además de su preparación propiamente académica, del dominio teórico-práctico de su respectivo campo disciplinario, así como de su preparación en la investigación para su creación y su promoción en la sociedad, requieren, como parte ineludible de su desarrollo profesional, de una formación más amplia, acorde a esas cualidades del desempeño humano que exigen esas condiciones actuales. Asimismo, las instituciones de educación superior, encargadas de la formación de esos profesionales, están llamadas a jugar un papel activo en las transformaciones y acciones que posibiliten la supervivencia humana y el afrontamiento y superación de esa problemática. La UNESCO (1998) declaró, de manera general, que la educación universitaria debe ser el 
motor del desarrollo económico, social y cultural de un país, debe contribuir al desarrollo sostenible y al mejoramiento de la sociedad en su conjunto; y puntualiza los que señala como sus principales encargos sociales: a) ser permanente y estar abierta a los distintos ámbitos de la vida; b) contribuir a la solución de los problemas del desarrollo que le plantea la sociedad; c) establecer el nexo con los demás sistemas educativos e impulsar la investigación en su vínculo con la formación; y d) mantenerse como el principal instrumento de la cultura y de la generación tecnológica.

\section{Desafíos en torno}

\section{a los avances científico-técnicos y del conocimiento}

Otras condiciones que representan desafíos sociales para la educación superior tienen que ver con la permanente producción y enorme acumulación de conocimiento en las distintas áreas, y con los grandes avances en las tecnologías de la información y la comunicación. Esta situación implica que la práctica de la enseñanza, ya desde hace tiempo cuestionada pero aún no del todo superada en la realidad, que conduce al aprendizaje con base en la memorización para fines de su reproducción en un examen, resulta inviable y obsoleta y no se ajusta a esas exigencias que impone el mundo actual (Fariñas, 2004; Cuevas, 2013a). Significa entonces que, entre otras cuestiones, la formación superior del estudiante debiera estar centrada, en vez del aprendizaje de contenidos que lo hacen pasivo y reproductor, en el desarrollo de estrategias y habilidades de aprendizaje, que le permitan constituirse en un sujeto dinámico, activo, creativo y propositivo; algunas de dichas habilidades que señala Fariñas (2004) serían: a) la habilidad de comprensión (de un texto, de sí mismo, de los demás, de su mundo, de su objeto disciplinario de estudio, etc.); b) la habilidad de búsqueda, creación y comunicación del conocimiento; c) la habilidad de formulación de problemas (de sí mismo, de su mundo, de su objeto disciplinario de formación profesional, etc.) y no sólo de solución de problemas; asimismo d) la habilidad de autovaloración y autorregulación.

De manera más específica resaltan los resultados de investigaciones recientes sobre las implicaciones del acceso masivo a las tecnologías de la informática y la comunicación (TIC) en la formación de nuevos sujetos denominados como generación "NET", caracterizados por: preferencias hacia los ambientes ricos en imágenes multimedios en vez del texto, involucración activa en tareas en vez de sólo lectura o disertación y escritura, trabajo en grupo colaborativo, multitarea que implica la actuación a la vez en distintas actividades como oír música, trabajo en la computadora, chatear, etc. (Lara y cols., 2009); la investigación plantea la necesidad de un giro en las prácticas docentes que contemple esas nuevas necesidades y condiciones. El mérito de esta investigación es poner en tela de juicio los criterios tradicionales de la docencia eficiente, que ya no corresponden a las necesidades y modos de ser y hacer de los alumnos de hoy. Desde luego, ya se ha avanzado en la incorporación de las tecnologías de la informática y la comunicación en la educación, pues se han generado múltiples trabajos de investigación, unos orientados a la creación de nuevas técnicas de ese tipo, otros para estudiar las ventajas o desventajas de su uso o las implicaciones en el rendimiento y desempeño escolar, o bien como recurso para ampliar el acceso a la educación, o en general para el mejoramiento de la calidad educativa. Sin embargo, hasta ahora la tendencia generalizada en este campo no ha trascendido los límites de los fundamentos y prácticas de orientación tradicional de la educación, pues sólo se han situado en el reto de hacer más eficientes dichas prácticas a través de su digitalización, de modo que aún está pendiente el reto de una educación integral del estudiante (Cuevas, 2013b).

La formación profesional con base en todas esas características y consideraciones, implica la reformulación y reestructuración de los planes de estudio; la formación en estrategias de aprendizaje requiere también de una enseñanza con base en 
estrategias afines, aprovechando e incorporando los nuevos conocimientos y tecnologías para la formación de un profesional de su tiempo, que esté completamente habilitado para valorar y enfrentar los nuevos retos que va generando el desarrollo de cada sociedad y de la humanidad en general, principalmente en relación con su campo disciplinario, pero también y de manera más global con otras áreas profesionales. Relacionado con esta cuestión, la UNESCO (1998) considera como los pilares formativos que la educación superior requiere fomentar o reforzar: a) "aprender a conocer", referido a la apropiación de las herramientas conceptuales para conocer y comprender la realidad desde la estrategia de aprender a aprender; b) "aprender a hacer" que implica el dominio de los métodos, técnicas y modos de actuación profesional para afrontar los retos relacionados con el campo de formación; y c) "aprender a convivir", a través de la relación con los demás, el trabajo en equipo, el intercambio social de manera pacífica, la actitud de colaboración y la aceptación de las diferencias.

\section{Desafíos de la estructuración interna del proceso formativo y del acceso a la educación superior}

Bajo este rubro se pueden explicitar algunas cuestiones y exigencias actuales que, de alguna manera, tendrían que considerarse para orientar la nueva formación profesional que se plantea; entre ellas están, principalmente, las siguientes: a) con respecto a la evidente discontinuidad entre los estudios universitarios y los que le preceden, resulta imprescindible una reestructuración de la educación superior, vinculándola a los estudiantes y profesores de todos los niveles, principalmente los bachilleratos, de modo que se genere la dinamización en la negociación y el consenso sumando fuerzas favorables al interés general y al bien común; $b$ ) también representa una exigencia la continuación del avance hacia el establecimiento y concreción de los vínculos interdisciplinarios y, asimismo, hacia el sustento de la educación superior en el principio de aprender a aprender y los sistemas universitarios en sistemas de multiuniversidades articuladas, respetuosas de la autonomía de sus integrantes y de las redes que establezcan sus grupos de docencia e investigación (González, 2005; De Sousa, 2005); c) el reto de la orientación educativa y profesional del estudiante, es la superación de su reducción al monitoreo para mejor eficientizar la reproducción mecánica del conocimiento y/o la obtención de las notas o calificaciones como sistema para la evaluación del desempeño académico que establece el sistema, hacia el logro del desarrollo como sujeto de formación profesional, que se construye un sentido, una implicación y un compromiso personales con la formación en su disciplina, autovalorándose, autorregulándose y planteándose metas a futuro en relación con su profesión (González, 1999; 2004); desde luego, no se pueden desconocer algunas voces y perspectivas a lo interno de algunos planes de estudio en algunas instituciones y países, que plantean superar esa visión tradicional del quehacer educativo, considerando el carácter activo del proceso enseñanza-aprendizaje que, por lo tanto, requiere de la implicación participativa y motivada de los alumnos como sujetos que construyen el conocimiento y el saber, y que también supone la superación de la evaluación como repetición de información, sin embargo, el avance en esa orientación sigue siendo muy escaso, de manera que su necesaria ampliación y consolidación representa un reto más de la educación superior; d) representa también un desafío la elevación de los índices de graduación considerando niveles superiores de eficiencia y retención con base en conceptos nuevos de equidad y permanencia; e) asimismo, la mejora en la motivación profesional de los estudiantes con respecto a su disciplina, que les posibilite inmiscuirse más allá de los lineamientos y criterios formalmente establecido en los planes de estudio, así como desarrollar su creatividad en la búsqueda y producción del conocimiento y convertirse en agentes de su formación y desempeño profesional con mayor eficiencia, calidad y compromiso social (González, 2009; Domínguez, 2006); y f) las con- 
diciones de la sociedad de ahora, considerada "la sociedad del conocimiento", han contribuido al incremento en la demanda de educación superior; la asunción de este desafío implica la superación de la perspectiva inmersa en algunos discursos y políticas educativas, al plantear que la masificación educativa constituye un impedimento para una educación de calidad, y ahí uno de los fundamentos esgrimidos para las prácticas selectivas de ingreso a la educación superior; en realidad no hay sustento de esa oposición, pues cada vez se puede formar con calidad a más estudiantes si la orientación es la búsqueda del rigor, la exactitud, la claridad, la eficacia y la eficiencia, a través de su formación en la crítica, en el análisis, en el diálogo, en la exposición verbal y escrita, en la búsqueda de un sentido de su vida, de su papel en su localidad, en su país y en su mundo (González, 2001). Inclusive, a partir de una postura más crítica al respecto, se hace posible concebir la interrelación de ambas cuestiones, planteando que la calidad de la educación superior sólo tiene sentido si es accesible a los sectores sociales más amplios; sin la masividad no es posible la calidad educativa porque se carece del impacto y de la significación social que requieren las transformaciones sociales más trascendentes. Asimismo, en el afrontamiento de este desafío la educación superior tendrá que considerar, fundamentalmente, cómo garantizar la masiva igualdad de oportunidades de acceso a la educación universitaria, entre otras cuestiones más allá de la única idea de dar cabida a todos en los tradicionales espacios universitarios y conforme a las mismas modalidades formativas, para tener en cuenta la amplia gama de posibilidades y alternativas espaciales, de modalidades y de recursos técnico-científicos que se encuentren disponibles, o de otros nuevos que sea posible generar.

\section{Desafíos en torno a la formación de valores en los estudiantes}

De manera generalizada el concepto de valor hace referencia a realidades con una significación social positiva en determinado contexto sociocultural que funge, interrelacionadamente, como medio de conocimiento y como medio de regulación y orientación del comportamiento; esto es, implica una función cognitiva y una función de control respectivamente, que hacen posible la evaluación de la realidad y de sí mismo (Colectivo de autores, 2003).

Los valores se forman de manera dialéctica, a través de "las contradicciones que ocurren entre las nuevas aspiraciones, necesidades y puntos de vista sobre la realidad que surgen en el proceso histórico, y las viejas formas o vías de satisfacción de las necesidades y de realización de las expectativas que no dan respuesta a las nuevas necesidades y aspiraciones" (Colectivo de autores, p. 20); se trata de contradicciones entre nuevos valores y los instituidos que frenan el desarrollo, contradicciones entre lo individual y lo social y contradicciones ante los cambios bruscos en el sistema de valores con los que los sujetos se identifican y/o con los que construyen sus proyectos de vida y de realización personal; estas últimas contradicciones suelen ser la fuente de la crisis de valores, es decir, de un vacío moral que puede conducir al nihilismo moral, a la pérdida del sentido de la vida y hasta de la propia existencia. La crisis de valores se asocia a procesos de distorsión o deterioro de la subjetividad que se expresa en dependencias y adicciones, en prácticas de violencia, drogadicción, aberración sexual, etc.

Desde el nivel psicológico el ser humano es productor de valores en tanto agente creador de la riqueza material y espiritual que a la vez se convierte en fuente de su desarrollo. El desarrollo moral se constituye en producto del proceso de interiorización de normas y reglas morales, desde donde ocurre la formación de determinadas instancias morales "internas" que, como sistema de formaciones psicológicas, regulan desde un plano interno la conducta del sujeto. En otras palabras, los valores son formaciones subjetivas relativamente estables que, al movilizar y regular la actuación del sujeto de determinada manera, se convierten en motivos de la personalidad; su contenido se corresponde con las significaciones sociales positivas y se constituye a través de la implicación personal y motivada de la persona en la interacción social. 
En congruencia con estos planteamientos el ser humano no nace moral o axiológico, sino que se forma a través de su participación dinámica en los distintos contextos sociales de implicación dentro de su medio social, constituyéndose y legitimándose como miembro de su grupo social o comunidad de práctica (Dreier, 1999; Lave y Wenger, 2003); inicia en el contexto familiar y después se extiende a los otros ámbitos como la escuela, las organizaciones laborales, los clubes deportivos, los grupos informales, etc.

Aunque la escuela, desde la básica hasta la superior, se considera la institución por excelencia para la educación de los sujetos que en ella se insertan, la práctica tradicional en este contexto se ha reducido a otorgar prioridad al nivel instructivo y en la esfera cognitiva, y muy poco o nada se considera la cuestión más amplia de educación, proceso que tiene que ver con la unidad afectivo-cognitiva, con la formación de valores como convicciones personales que regulan y autorregulan la actuación de las personas. Los siguientes señalamientos, ampliamente reconocidos, son algunos de los fundamentos de esta aseveración: a) la dinámica de la escuela en sus distintos niveles se ha centrado en la enseñanzaaprendizaje de contenidos académicos, principalmente de manera mecánica y para ser repetidos en exámenes a través de los que se mide el rendimiento escolar expresado en una nota o calificación que, entre otras cuestiones, se convierte en criterio de comparación, de jerarquización de los desempeños e incluso de discriminación; b) con relación a las normas y reglamentos, ellos suelen aplicarse de manera impositiva o, a lo sumo, se intenta que los sujetos adquieran los significados correspondientes, pero no existe el diseño e implementación de una dinámica escolar de implicación motivada y comprometida de los sujetos, de manera que las normas $\mathrm{y}$ valores adquieran un sentido personal y se conviertan así en reguladores y autorreguladores de las acciones de los sujetos; c) en correspondencia con lo anterior, la regulación del comportamiento de los estudiantes sucede por controles externos, como la calificación, las sanciones, la opinión de los otros; en ese sentido también, el quehacer de la escuela se asume, por parte de los estudiantes, como una obligación que hay que cumplir, incluso muchas veces como algo que hay que soportar y, en correspondencia, siempre se buscan maneras de cometer fraudes, como copiar en el examen, "portarse bien" sólo cuando está presente la autoridad, cumplir para satisfacer al profesor o a la familia, bajar de internet lo que debía ser un trabajo de elaboración personal, lo que representa una actitud de no implicación y de mínimo esfuerzo, etc. Esa perspectiva tradicional sólo propicia, cuando más, que los sujetos conformen su moral por lo que ella significa para otros más que para sí mismos, concediendo prioridad a la regulación por sobre la autorregulación, lo cual conduce a una doble moral: incongruencia entre el discurso verbal y el comportamiento moral efectivo, o distanciamiento entre lo que se siente, se piensa y se hace (López, 2004).

En suma, esa formación integral de los estudiantes de educación superior, que implica su formación en valores y que les permite redimensionar su quehacer como sujetos de formación profesional, en su relación con las significaciones sociales positivas que se construyen en sus contextos locales y social general como país y como humanidad, máxime en esta época catalogada de "crisis de valores", representa un enorme desafío que, de manera apremiante, requiere considerar en la actualidad la educación superior.

\section{Conclusión}

Los grandes desafíos que caracterizan la época actual condicionan exigencias fundamentales que demandan su atención en la estructuración formativa de la educación superior; esto implica la apremiante necesidad de construir lineamientos teóricoconceptuales y orientaciones prácticas para elaborar e implementar las propuestas de formación profesional. Hacia ese cometido se hace imprescindible, de manera general, las siguientes orientaciones.

Primero, ajustar los planes de estudio considerando los avances científico-técnicos como la informática y la comunicación; asimismo, este proceso implica la superación permanente de la planta 
docente, la actualización y evaluación continua de los programas de formación profesional, de manera que sean pertinentes a las necesidades sociales de producción y del desarrollo sustentable.

Segundo, la democratización para un acceso masivo y con calidad y la integración universitaria dentro de cada país y entre países. Es necesario superar la inequidad en el acceso a la educación superior, así como las comparaciones entre escuelas o instituciones que ignoran las especificidades de contextos y las diferencias individuales, a veces abismales, de los estudiantes.

Tercero, encausar los esfuerzos para una formación profesional que supere el nivel puramente instructivo hacia un proceso de unidad educación-instrucción. La calidad educativa no debe confundirse solamente con el aprendizaje y aplicación de conocimientos y la sobrevaloración de unos sobre otros, ni con el éxito laboral conforme a los valores de competencia del sistema; la educación de calidad implica la estimulación a ser cada vez mejores y a comprender que cada uno está necesitado de los demás; implica la formación de un hábito de autoexigencia pautada por la solidaridad con los otros; asimismo, junto al conocimiento científico que generalmente busca explicaciones, tendría que considerarse el "conocimiento cultural" hacia la búsqueda y construcción de significados.

Cuarto, la formación y la práctica profesional sustentadas en valores para un desempeño ético, responsable y socialmente comprometido. Este desafío implica superar el nivel puramente racional del deber ser, pues no se trata sólo del conocimiento de normas, reglas y maneras de comportarse, sino su formación como convicciones personales que permitan regular y autorregular las actuaciones, como un proceso indisoluble entre pensar-sentir-actuar que necesariamente ocurre en la interacción con los otros, de acuerdo al contexto sociocultural de que se trate.

\section{Referencias Bibliográficas}

Colectivo de autores (2003). La educación de valores en el contexto universitario. La Habana: Editorial Félix Varela.

Cuevas, J. A. (2004). Algunas consideraciones en torno a la perspectiva tradicional del fracaso escolar. Revista Cubana de Psicología, 21, 101-105. Cuba: Universidad de la Habana.

(2013). Los alumnos ante el aprendizaje, el conocimiento y el desempeño escolar. En: C.L. Saucedo, C. Guzmán, E. Sandoval y J.L. Galaz (coords.), Estudiantes, maestros y académicos en la investigación educativa. México: ANUIES-COMIE.

Domínguez, G.L. (2000). "Identidad, valores y proyecto de vida". Artículo en soporte electrónico. Facultad de Psicología, Universidad de la Habana.

Dreier, O. (1999). Trayectorias personales de participación a través de contextos de práctica social. Psicología y Ciencia Social, 3(1). México: Universidad Nacional Autónoma de México Campus Iztacala.

Fariñas, L. G. (1995). Maestro, una estrategia para la enseñanza. La Habana: Editorial Academia. (2004). Maestro. Para una didáctica de aprender a aprender. La Habana: Editorial Pueblo y Educación.

Giddens, A. (1995). Modernidad e identidad del yo. Barcelona: Edit. Península.

González, F. (1987). Motivación profesional en adolescentes y jóvenes. La Habana: Editorial Pueblo y Educación.

(1993). Motivación moral en adolescentes y jóvenes. La Habana: Editorial Científico técnica.

González, M.V. (1999). La orientación profesional en la educación superior (alternativa teórico-metodológica para su implementación en los centros universitarios). Centro de Estudios para el Perfeccionamiento de la Educación Superior (CEPES), Universidad de la Habana.

González, C.P. (2001). La universidad necesaria en el siglo XXI. México: Editorial Era. 
(2005). Las nuevas ciencias y las humanidades. Barcelona: Ántropos Editorial.

Lara, B. A; Aguiar, B.M.E; Cerpa, C. G y Núñez, T. H. (2009). Relaciones docente-alumno y rendimiento académico. Un caso del Centro Universitario de Ciencias Exactas e Ingenierías de la Universidad de Guadalajara. Revista Sinéctica, 33, 1-15. México: Universidad de Guadalajara.

Lave, J., Wenger, E. (2003). Aprendizaje situado. Departamento editorial, FES Iztacasla UNAM.
López, B. L. (2004). De qué ética hablamos. La Habana: Editorial Félix Varela.

Organización de las Naciones Unidas para la Educación la Ciencia y la Cultura (1992). "Educación y transformación productiva con equidad". Santiago de Chile. (1998). Declaración mundial sobre la educación superior para el siglo XXI. París.

Sotolongo, P. (2006). Teoría social y vida cotidiana. La sociedad como sistema dinámico complejo. La Habana: Publicaciones Acuario, Centro Félix Varela. 